\title{
Study on Microstructure and Mechanical Properties of Low Cost Ti-Fe-B Alloy
}

\author{
Yangyang Sun ${ }^{1,2,3}$, Hui Chang ${ }^{1}$, Zhigang Fang ${ }^{4}$, Yuecheng Dong ${ }^{1,3,4^{*}}$, Zhenhua Dan ${ }^{1,4}$, Yanhua Guo ${ }^{1}$, Lian Zhou $^{1}$ \\ (1. College of Materials Science and Engineering/Tech Institute for Advanced Materials, Nanjing Tech University, Nanjing 210009, China)
}

(2. Jiangsu Collaborative Innovation Center for Advanced Inorganic Function Composites, Nanjing 211899, China)

(3. State Key Laboratory of Metal Material for Marine Equipment and Application, Anshan 114000, China)

(4. Naval Research Academy, Beijing 100000, China)

Abstract: Microstructure evolution and mechanical properties of low cost Ti-2Fe-0.1B alloy under different heat treatment were studied. Results indicated that two kinds of equiaxed microstructures with different characteristics were obtained in conventional and double annealing, and typical lamellar microstructure was obtained in $\beta$ annealing. Tensile test results shown that as-received rolled alloy possess highest strength and plasticity simultaneously due to fine and entangled microstructure. Uniform equiaxed dimples were observed in microstructure, which revealed ductile fracture morphology.

Key words: titanium alloy; microstructure; heat treatment; mechanical properties

\section{$\underline{\text { 1. Introduction }}$}

Titanium and titanium alloys are a kind of "marine metal" with high specific strength, non-magnetic properties and excellent corrosion resistance in seawater. They have been widely used in many fields like deep sea equipment, seawater desalination and ships ${ }^{[1-3]}$, providing an effective material support for the safety and reliability of offshore engineering equipment ${ }^{[4]}$. However, due to the high cost of manufacture and the difficulty in processing, the usage rate of titanium alloy is still limited. Therefore, it is crucial to find an effective way to reduce the cost of titanium and titanium alloys and improve their properties at the same time. Adding 1.0 wt.\% Fe to the as-cast Ti- $10 \mathrm{Zr}$ alloy can significantly refine the width of the martensite $\alpha^{\prime}$ in the structure, which can increase the strength and hardness of the alloy, and improve the wear properties of the material ${ }^{[5]}$. This shows that it is effectively to improve the properties of titanium alloy by adding inexpensive alloying elements (Fe, etc.) However, most studies on the influence of Fe element to the properties of alloys are based on binary or multi-component titanium alloys. W F. Ho et al. ${ }^{[6]}$ studied the effect of Fe on mechanical properties and deformation behavior of $\mathrm{Ti}-5 \mathrm{Cr}$ alloy by adding different contents of $\mathrm{Fe}$. The results showed that the bending strength of $5 \mathrm{wt} . \% \mathrm{Fe}$ and $3 \mathrm{wt} . \% \mathrm{Fe}$ was higher than that of original alloy and other content alloys, and the bending strength was $2.2 \sim 2.5$ times higher than that of CP-Ti. L. Bolzoni et al. ${ }^{[7]}$ made a new kind of low-cost Titanium Alloys by mixing titanium with stainless steel, and found that their properties were comparable to those of forged and powder metallurgical titanium alloys. D J. Lin et al. ${ }^{[8]}$ studied the quenching of Ti-7.5Mo alloy with different Fe content and found that Ti-7.5Mo-2Fe alloy exhibited good mechanical behaviors. However, Ti-7.5Mo-1Fe alloy is prone to brittle fracture due to low strength and low ductility. The main reason is that $0.5 \sim 2 \mathrm{wt} . \% \mathrm{Fe}$ is added to the titanium alloy, which will form an adiabatic $\omega$ phase in the alloy ${ }^{[9]}$. Although the appearance of the $\omega$ phase can increase the strength and elastic modulus of the alloy to some extent, it can also reduce the plasticity. B. Sengupta et al. ${ }^{[10]}$ studied a new low-cost titanium alloy Ti-22Fe-4Si-4Cr. The compressive strength of the alloy can reach $2.56 \mathrm{GPa}$, and the as-cast alloy can maintain good hardness (about $710 \mathrm{HV})$. Y. Zhang et al ${ }^{[11]}$ studied the effect of different Fe content on microstructure and mechanical properties of as-cast Ti-xFe-B alloy. It was found that with the increase of Fe content, the hardness and tensile strength of Ti-xFe-B alloy were improved. When the Fe content was $3 \sim 4$ wt.\%, the alloy could achieve the best matching of strength and plasticity. These studies show that appropriate or trace of Fe addition can play a positive role in improving and optimizing the properties of titanium alloys. It can not only improve the cold and hot deformation ability of the alloys, but also have a very important influence on the performance optimization of the alloys, especially on the matching of strength and toughness.

In terms of organizational microstructure, $\alpha+\beta$ titanium alloy has good plastic matching and good welding performance, and is more suitable for application on ships. In this paper, the microstructures and mechanical properties under different heat treatment of low cost Ti-2Fe-0.1B alloy, on the basis of the study on low-cost Ti-Fe-B titanium alloy ${ }^{[11]}$ in the early research. At the same time, it can further enrich the study on the development of low-cost titanium alloys containing Fe and B elements and provide relevant theoretical support.

\section{Material and experiments}

The Ti-2Fe-0.1B alloy used in this study was received in a hot-rolled bar with a diameter of $\Phi 20 \mathrm{~mm}$ from which cuboid specimens $(\Phi 10 \mathrm{~mm} \times 10 \mathrm{~mm}$ for microstructure analysis and $\Phi 5 \mathrm{~mm} \times 4 \mathrm{~mm}$ for X-ray diffraction analysis) were spark machined. The transus temperature ( $\left.\mathrm{T}_{\beta}\right)$ of the alloy obtained by examination of microstructure is $(857 \pm 5)^{\circ} \mathrm{C}$ and the chemical compositions of the material are provided in Table 1 . In order to obtain different microstructures of the alloy, three different heat treatment were selected based on the $\beta$ transus temperature $\left(\mathrm{T}_{\beta}\right)$, including conventional annealing (CA,720 $\left.{ }^{\circ} \mathrm{C} / 1 \mathrm{~h} / \mathrm{AC}\right)$, double annealing $\left(\mathrm{DA}, 830^{\circ} \mathrm{C} / 1 \mathrm{~h} / \mathrm{AC}+520^{\circ} \mathrm{C} / 4 \mathrm{~h} / \mathrm{AC}\right)$ and $\beta$ annealing $\left(\beta \mathrm{A}, 883^{\circ} \mathrm{C} / 1 \mathrm{~h} / \mathrm{AC}+520^{\circ} \mathrm{C} / 4 \mathrm{~h} / \mathrm{AC}\right)$. The microstructure analysis, tensile mechanical test, $\mathrm{X}-\mathrm{ray}$ diffraction $(\mathrm{XRD})$ test and scanning electron microscope (SEM) observation of fracture morphology about the alloy after heat treatment were carried out.

Tab.1 Chemical composition of Ti-2Fe-0.1B alloy (mass fraction, wt\%)

\begin{tabular}{cccccccc}
\hline Alloy & $\mathrm{Fe}$ & $\mathrm{B}$ & $\mathrm{C}$ & $\mathrm{H}$ & $\mathrm{O}$ & $\mathrm{N}$ & $\mathrm{Ti}$ \\
\hline Ti-2Fe-0.1B & 1.89 & 0.08 & 0.014 & 0.0012 & 0.062 & 0.004 & Bal. \\
\hline
\end{tabular}

The Ti-2Fe-0.1B alloy bar after heat treatment was processed into cylindrical tensile specimens. The dimension of parameters which tensile specimens include is described on Fig 1. The tensile tests were executed on the ETM205D stretcher at room temperature separately. The phase identification of the alloys was analyzed by X-ray diffractometry (XRD, DMAX-RB, Rigaku, Japan) using $\mathrm{Cu}$ K $\alpha$ radiation $\left(\lambda=0.15406 \mathrm{~nm}\right.$ ) with scanning speed at $4 \% \mathrm{~min}$ and scanning range $2 \theta$ between $10 \sim 90^{\circ}$. Microstructure

(C) The Authors, published by EDP Sciences. This is an open access article distributed under the terms of the Creative Commons Attribution License 4.0 (http://creativecommons.org/licenses/by/4.0/). 
observations were conducted by an optical microscopy (OM: ZEISS, A1M) and the scanning electron micros-copy (SEM). Metallographic examinations of the alloys were conducted after polishing and etching with a reagent $\left(10 \% \mathrm{HF}+20 \% \mathrm{HNO}_{3}+70 \% \mathrm{H}_{2} \mathrm{O}\right)$ for about $6 \mathrm{~s}$. Fractographic observation was indispensable in analyzing fracture feature and mechanism.

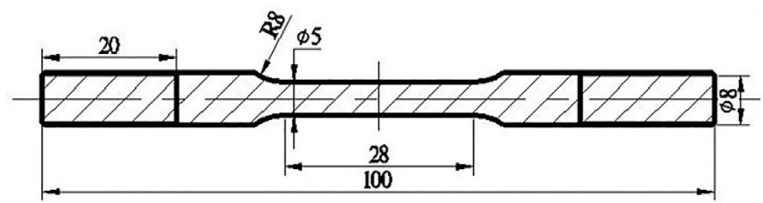

Fig.1 Sizes of the specimen for tensile test

\section{$\underline{\text { 3. Results }}$}

\subsection{Microstructures}

Representative microstructure of Ti-2Fe-0.1B alloy after different heat treatments are shown in Fig. 2. It can be seen the microstructure of as-rolled Ti-2Fe-0.1B alloy exhibits a fine and twisted $\alpha$ phase (Fig.2a), which is disorderly and distributed with obvious boundaries. The grain of microstructure taken from CA specimens is growing in the direction of the equiaxed. The grain size is obviously larger than the rolled, and the $\alpha$ phase grows apparently in an irregular state, but the $\alpha$ phase and the $\beta$ phase can be clearly distinguished (Fig.2b). After DA treatment, primary $\alpha$ turned into blocky $\alpha$ around by a small amount of transformed $\beta$, exhibiting equiaxed microstructure characteristics with the continually spheroidization process (Fig.2c). With the temperature increasing, the size of $\alpha$-colony increases and the morphology also shows difference. The volume fraction and size of each phase in the microstructure were measured by Pro-imaging and Image Pro Plus (IPP) professional image analysis software. The average grain size of the alloy treated by DA is about $10.08 \mu \mathrm{m}$ and the content of primary $\alpha$ lamellar increases, but the equiaxed primary $\alpha$ phase still occupied a higher proportion with about $64.31 \%$ volume fraction. The Fig.2d shows that the typical lamellar microstructure composed of the transformed $\beta$ and the coarse-grained $\alpha$ lamellar obtained by the treatment of $\beta$ A. The original $\beta$ grain boundary is retained with about $1 \sim 3.5 \mu \mathrm{m}$ thickness of the crystal grains containing $\alpha$-colony, and there are obvious $\alpha$ phase (grain boundary $\alpha$ ) and lamellar $\alpha$ in the microstructure.

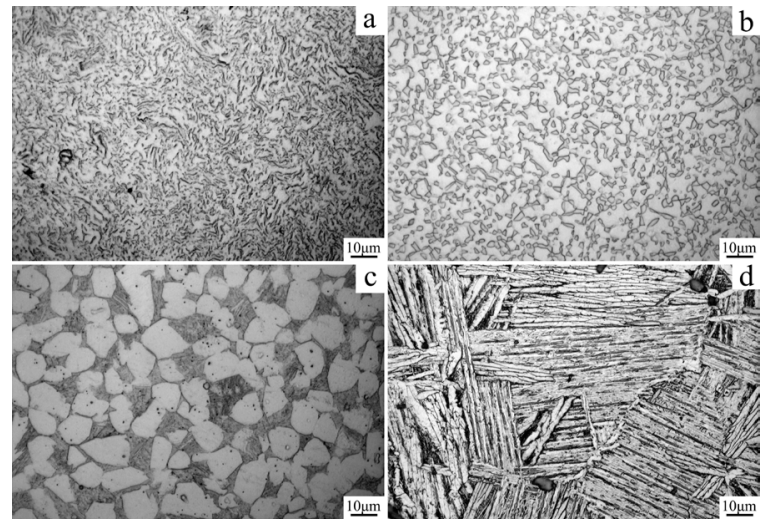

Fig.2 The microstructure of Ti-2Fe-0.1B alloy at different heat treatment: (a)Rolled, (b)CA, (c)DA, (d)BA

The X-ray diffraction pattern of theTi-2Fe-0.1B alloy shown in Fig. 3 illustrates the Ti-2Fe- $0.1 \mathrm{~B}$ alloy is an $\alpha+\beta$ two-phase titanium alloy. In the $\mathrm{X}$-ray diffraction pattern of the alloy, the characteristic 1 peak of the $\beta$ phase is offset from the standard card. The main reason is that the Fe element added to the alloy can induce the distortion of lattice. It can be concluded from the content of $\alpha$-phase and $\beta$-phase in Ti-2Fe- $0.1 \mathrm{~B}$ alloy before and after heat treatment quantitatively analyzed by XRD pattern of specimens after $\beta$ A processing, the primary $\alpha$ phase is basically transformed into $\beta$ phase. However, in the process of cooling, the secondary $\alpha$ phase were generated, so that the content of the $\alpha$ phase about $89.71 \%$ can maintain at a high level. Since the content of Fe and B elements in ti-2fe- $0.1 \mathrm{~B}$ alloy is relatively low, there is no diffraction peak of TiB and TiFe alloy compounds in the X-ray diffraction pattern. 


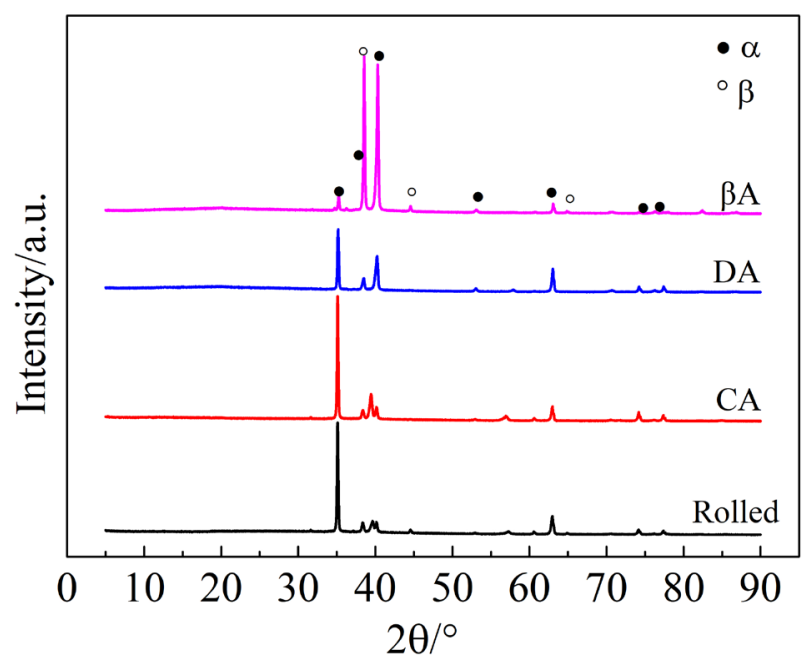

Fig.3 X-ray diffraction patterns of Ti-2Fe-0.1B alloy after rolling and heat treatment

\subsection{Tensile properties}

The stress-strain curve and tensile properties of Ti-2Fe-0.1B alloy at room temperature are shown in Fig. 4 and Table 2 respectively. It can be seen the as-rolled specimen has the highest tensile strength (444.36 MPa) with the best elongation (about $25.17 \%$ ) and that of the DA processing is the worst (296.20 MPa). In general, the properties of the as-rolled specimen can achieve the best match between strength and plasticity because of the effect of Fe element on grain refinement. The performance of the lamellar microstructure obtained by $\beta$ A processing is poor, mainly because the $\beta$ A processing is performed above the $\beta$ transus temperature, which tends to make the $\beta$ crystal grain or the grain boundary become coarse, making the crack easily grow at the grain boundary and resulting in the decrease of strength and plasticity ${ }^{[12]}$. The strength of Ti-2Fe- $0.1 \mathrm{~B}$ alloy after heat treatment is lower than that of the as-rolled, which is dependent on the microstructure and phase content obtained by heat treatment. As can be seen from Fig.4, during the tensile test, the equiaxed microstructure has a relatively obvious necking phenomenon, which is caused by the deformation coordination of equiaxed primary $\alpha$ phase in the equiaxed microstructure ${ }^{[13,14]}$. The lamellar microstructure enters the yielding stage prematurely because the large $\alpha$-colony can reduce the strength of the lamellar layer. And the presence of $\alpha$-colony strongly can hinder the slip progress, and the dislocation does not easily pass through the $\alpha / \beta$ phase interface, and stress concentration occurs at the phase interface, causing the specimen to enter the yield stage prematurely ${ }^{[15]}$. The reason why the strength of equiaxed microstructure obtained by DA processing does not increase was that the content of equiaxed primary $\alpha$ phase in the alloy decreased with the increase of temperature compared with the CA specimen. Although the precipitation of secondary $\alpha$ phase from the transformed $\beta$ of the alloy can improve the strength of the alloy, the content of primary $\alpha$ phase in the alloy is reduced more with the obvious phenomenon of grain coarsening. Therefore, the decrease in strength caused by the decrease in the content of equiaxed primary $\alpha$ phase and the coarsening microstructure is significantly stronger than the increase in the strength caused by the secondary $\alpha$ phase, resulting in the decrease of mechanical properties and the increase of plasticity at room temperature ${ }^{[16]}$.

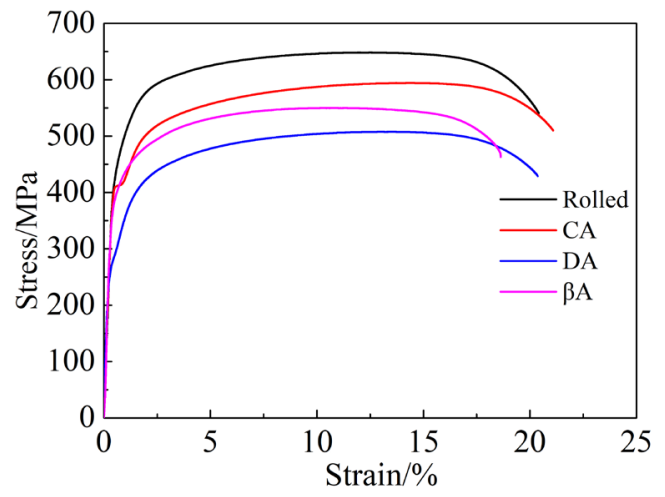

Fig.4 Stress-strain curves of Ti-2Fe-0.1B alloy after rolling and heat treatment

Table 2 Tensile properties of Ti-2Fe-0.1B alloy with different heat treatment at room temperature

\begin{tabular}{cccc}
\hline Heat-Treatment & $\mathrm{Rp} 0.2 / \mathrm{MPa}$ & $\mathrm{Rm} / \mathrm{MPa}$ & $\mathrm{A} / \%$ \\
\hline Rolled & 444.36 & 636.56 & 25.17 \\
CA & 415.30 & 596.91 & 21.80 \\
DA & 296.20 & 528.65 & 24.09 \\
BA & 390.64 & 550.71 & 26.73 \\
\hline
\end{tabular}




\subsection{Fractograph}

Generally, the alloy fails under the joint action of external stress and the environment, so the fracture morphology can record the whole process of crack formation, crack propagation and fracture in the alloy microstructure. It can be seen from Fig. 5 that the fracture morphology of the specimens with different treatment exhibit the characteristics of ductile fracture. There is obvious macroscopic plastic deformation near the fracture.There are only fibrous zone and the shear lip on the fracture surface, and there are numerous small peaks by the continuous propagation and interconnection of microcracks during plastic deformation in the fibrous zone caused. Among the four kinds of specimens, only the area of the shear lip in the microstructure by CA heat treatment is larger, which indicates that the plasticity of the alloy under this condition is poor. When the crack propagates outward from the fiber region at the center of the specimen, the entire outer region of the crack has a large plastic deformation, where the shear lip is formed. Some small facets like geometric shapes can be found on the fracture surface of the specimen after $\beta$ A treatment, which is related to the presence of coarse and large $\beta$ grain in the microstructure.

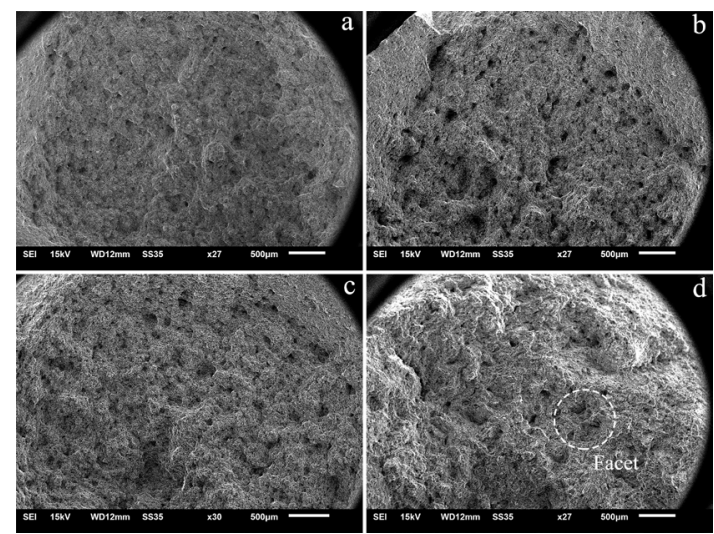

Fig.5 The macro fractures of the tensile specimens: (a)Rolled, (b)CA, (c)DA, (d)BA

Microscopic morphology of the fracture of the tensile specimens after heat treatments are shown in Fig.6. Many aggregated micropores can be seen in Fig. 6. As the tensile stress increases, the severe plastic deformation can cause the void nucleation at the fracture to gather, and grow under the shear stress into a new cavity. Fine equiaxed dimples are evenly distributed on the fracture surface for as-rolled and CA heat treatment, which indicates that the fracture mode is ductile fracture. There are tearing ridges in the fibrous region of four kinds of microstructures, due to severe plastic deformation. At the same time, the height of the tearing edge on the surface of the fracture is low, and the shape of shear dimple in the tearing ridges is also very clear. The dimples are formed at the second phase firstly, and the uniformly distributed particles make the dimples evenly distributed and exhibit good plasticity. During the stretching process, the accumulation of microscopic voids reduces the repulsive force of the dislocations in the alloy structure, and the continuation of the tensile stress causes the dislocations to move toward the newly formed microscopic voids, resulting in the growth of the voids ${ }^{[18]}$. In addition, the cross-sectional volume between the micropores decreases gradually, and the voids are interconnected to form an equiaxed dimple finally. From the micro-fracture morphology of the tensile specimens obtained by the $\beta$ A heat treatment, it can be seen fine dimples are distributed on the cleavage surface, and the overall distribution is uneven. Compared the fractures with other specimens, the dimples become larger and deeper, which can bring perfect plasticity for $\beta \mathrm{A}$ treated specimens.
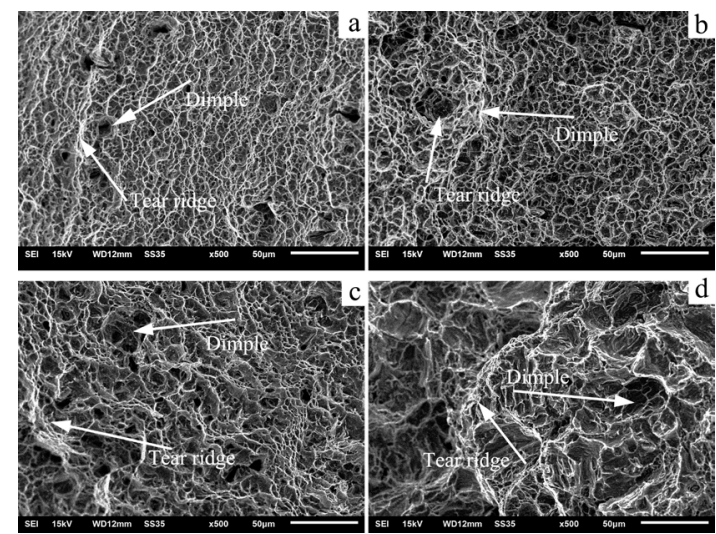

Fig.6 The fracture morphology of the tensile specimens: (a)Rolled, (b)CA, (c)DA, (d)BA

\section{Analysis and discussion}

The tensile properties of the as-rolled Ti-2Fe-0.1B alloy in this study are better than those of pure titanium (TA1, TA2) used in ships, and the plasticity also maintains a high level, due to fine and entangled microstructure. From the comprehensive observation and analysis on the plasticity and the tensile fracture morphology of the alloy at room temperature, it can be found that strength of specimens obtained by CA heat treatment is lower than that of other specimens. The main reasons are that coarse equiaxed $\alpha$ phases and precipitated lamellar $\alpha$ phases appeared in the microstructure by DA, which still maintains the morphology of equiaxed microstructure. The size of the $\alpha$-phase clusters with similar orientation gradually increases, and the thickness of secondary $\alpha$ phase in microstructure also becomes thicker. Clear $\alpha$ grain boundaries in the microstructure by DA can obviously promote initiation of cracks. From the fracture morphology of the alloy, it can be seen the fracture morphology is composed of dimples, 
which indicates that the fracture mode is ductile fracture. The size and depth of the dimples reflect the plasticity of the alloy to a certain extent. When the dimples are large and deep, the plasticity of alloy is perfect. When the dimples are small and shallow, the alloy is poorly plastic ${ }^{[19]}$. Under the $\beta A$ heat treatment, all the original primary $\alpha$ phases are transformed into lamellar $\alpha$ phases with the presence of coarse $\beta$ grains. Because of the existence of grain boundary $\alpha$, the fracture mode of specimen from intergranular fracture to intragranular fracture, which requires a large amount of energy to consume, improving the tensile properties. Microcracks are mainly formed at the primary $\alpha$ grain boundary, the $\alpha / \beta$ phase boundary or the $\beta$ grain boundary. On the other hand, the presence of the $\alpha$ lamellar can increase the number of changes in the direction of crack propagation, so that the crack propagation path is prolonged and more energy need to be consumed.

In addition to the reasons mentioned above, the presence of Fe also has an important effect on the microstructure and properties of the alloy. As an important alloying element of metastable $\beta$ titanium alloy, Fe can play a significant role in reducing the martensitic transformation temperature of titanium alloy, improving the processing properties of the alloy, and enhancing the strengthening effect of heat treatment, but the strengthening effect mainly depends on the difference of size between the matrix and the added atom. The atomic radius of Ti is $0.147 \mathrm{~nm}$, and that of Fe is $0.127 \mathrm{~nm}$, so the difference in size between the two elements is obvious. The total elastic modulus of the alloy varies with the addition of $\mathrm{Fe}$, and the slip resistance of the movable dislocations within the grain increases during the tensile deformation, so that the strength of the alloy can be improved ${ }^{[20,21]}$. The main reason for the decrease in the strength of the alloy after heat treatment is that the fine equiaxed microstructure is transformed into the equiaxed primary and lamellar $\alpha$ phase with coarse grains, resulting in the increase of grain boundaries.

\section{Conclusion}

1.The Ti-2Fe-0.1B alloy is an $\alpha+\beta$ titanium alloy with the high plasticity, which has the better tensile properties of the as-rolled alloy than that of pure titanium (TA1, TA2). The performance of the Ti-2Fe-0.1B alloy can be better matched to meet the requirements for marine equipment。

2.Fine and uniform equiaxed microstructure was obtained by conventional annealing, equiaxed microstructure with diameter of $9.92 \mu \mathrm{m}$ and volume of $64.31 \%$ was obtained by double annealing, and lamellar microstructure with coarse grain of $\beta$ phase was obtained by $\beta$ annealing.

3.After heat treatment, the strength of Ti-2Fe-0.1B alloy is obviously lower than that of the as-rolled. The main reason for the decrease in the strength of the alloy after heat treatment is that the fine equiaxed microstructure is transformed into the equiaxed primary and lamellar $\alpha$ phase with coarse grains, resulting in the increase of grain boundaries. The presence of $\mathrm{Fe}$ also has an important effect on the refinement of microstructure grains and properties of the alloy.

4.There are only fibrous zone and the shear lip on the four kinds of fracture surface, only the area of the shear lip in the microstructure by conventional annealing is larger, which indicates that the plasticity of the alloy under this condition is poor. Fine equiaxed dimples are evenly distributed on the fracture surface for as-rolled and CA heat treatment, which indicates that the fracture mode is ductile fracture.

5.Ti-2Fe-0.1B is a low-cost titanium alloy with high strength, and good plasticity, which has great research significance.

\section{Acknowledgements}

This work was financially supported by the Open Foundation of State Key Laboratory of Metal Material for Marine Equipment and Application (No. SKLMEA-K201807), China Postdoctoral Science Foundation (No. 2017M623392), the Program of Research and Practice Innovation for Postgraduates in Jiangsu Province (No. KYCX17_0984) and A Project Funded by the Priority Academic Program Development of Jiangsu Higher Education Institutions (PAPD).

\section{References}

[1] L. Li, J K. Sun, X J. Meng, Titanium Industry Progress. 21(2004) 19-24.

[2] W. Gao, C X. Zhang, Titanium Industry Progress. 25(2008) 6-10

[3] P G. Esteban, E M. Ruiz-Navas, L. Bolzoni, Metal Powder Report. 63(2008) 24-27.

[4] H. Chang, Beijing: Chemical Industry Press. 2017.

[5] W F. Ho, C H. Chen, C H. Pan, Materials Science and Engineering. C29(2009) 36-43.

[6] W F. Ho, C H. Pan, S C. Wu, Journal of Alloys \& Compounds. 472(2009) 546-550.

[7] L. Bolzoni, E. Herraiz, E M. Ruiz-Navas, Materials \& Design. 60(2014) 628-636.

[8] D J. Lin, J H. Chern Lin, C P. Ju, Materials Chemistry and Physics. 76(2002) 191-197.

[9] C H. Lian, S. Yang, H. Zhou, Hot Working Technology. 42(2013) 40-42.

[10] B. Sengupta, S. Shekhar, K N. Kulkarni, Materials Science and Engineering A. 696(2017) 478-481. 
[11] Y. Zhang, H. Chang, G Z. Li, Rare Metal Materials and Engineering. 46(2017) 180-184.

[12] Y. Chong, T. Bhattacharjee, J H. Yi, Scripta Materialia. 138(2017) 66-70.

[13] S K. Li, B Q. Xiong, S X. Hui, Transactions of Materials and Heat Treatment. 29(2008) 82-85.

[14] Lee D H, Nam S W, Choe S J, Materials Science and Engineering A. 291(2000) 60-67.

[15] X B. Liu, Y J. Zhai, T. Yu, Metallic Functional Materials. 25(2018) 25-30.

[16] S H. Luo, Q Y. Fen, H M. Xue, Transactions of Materials and Heat Treatment. 39(2018) 27-31.

[17] A X. Sha, X W. Li, J P. Chu, Chinese Journal of Rare Metals. 30(2006) 26-29.

[18] B Y. Leng, X B. Xian, X X. Pang, Rare Metal Materials and Engineering. 40(2011) 1470-1472.

[19] J W. Lei, W D. Zeng, Huan Wu, The Chinese Journal of Nonferrous Metals. 20(2010) 625-628.

[20] D M. Chen, S S. Huang, F. He, Titanium Industry Progress. 34(2017) 19-23.

[21] H. Wu, Y Q. Zhao, P. Ge, Rare Metal Materials and Engineering. 41(2012) 805-810. 